\title{
Inhibition of NOX2 contributes to the therapeutic effect of aloin on traumatic brain injury
}

\author{
Haihai Dong ${ }^{1^{*}}$, Haitao Wang ${ }^{2^{*}}$, Xuezhi Zhang ${ }^{2}$ \\ ${ }^{1}$ Emergency Department, Anqiu People's Hospital, Weifang, China, ${ }^{2}$ Department of Critical Care Medicine, Anqiu People's Hospital, \\ Weifang, China \\ *Haihai Dong and Haitao Wang contributed equally in this work.
}

\begin{abstract}
Traumatic brain injury (TBI) is a subset of brain injury induced by external mechanical forces to the head or neck. $T B I$ has been reported to be one of the leading causes of disability, and it causes a huge financial burden around the world. Aloin is the major anthraquinone glycoside extracted from Aloe species, and has presented anti-tumour, antioxidative and anti-inflammatory activities. However, few studies have focused the effect of aloin in treatment of TBI. Nicotinamide adenine dinucleotide phosphate oxidase (NOX) is the only subset of enzymes which produces solely the reactive oxygen species (ROS). A recent study showed that activation of NOX might aggravate the primary TBI, and among these members, NOX2 is the key member in regulation of uncontrolled ROS expression, and thus plays a critical role in development of inflammatory diseases. Here, we noticed that inhibition of NOX2 combined with aloin treatment promoted the recovery of brain function in a mice model as well as the viability rate in a cell model. A further study found that the inflammation response process was also inhibited after treatment. Then, we found that these effects might be mediated by the PI3K/AKT/mTOR signalling pathway and NOX2 might be a therapeutic target for TBI.
\end{abstract}

Key words: TBI, NOX2, aloin, PI3K/AKT/MTOR, apoptosis.

\section{Introduction}

Traumatic brain injury (TBI) leads to approximately 2.8 million cases of death and disability around the world, and remains to be the main cause of young adults deaths in the United States $[21,28]$. TBI is a disease mainly caused by an external mechanical force, leading to the neurological dysfunction and even long-term cognitive, psychological and social defects [22]. Insulin-like growth factor-1 (IGF-1) is critical for the growth and development of brain and is involved in response to central and peripheral nervous system damage [8]. Aloin is the major compound of aloe species, and plenty of studies have proved the anticancer, anti-viral and anti-inflammatory effects of aloin [2]. Recent studies found that treatment of aloe vera inhibited the inflammation response process in cell and mice models after stimulation of 
lipopolysaccharide (LPS), besides, the secretion of cytokines and production of reactive oxygen species (ROS) was also inhibited [24,34]. Another study has shown that compounds of aloe species inhibit the production of pro-inflammatory cytokines and nitric oxide via inhibition of the MAPK signalling pathway [15]. More studies proved that treatment of aloin decreased the high production of interleukin (IL)-8 induced by IL-1 $\beta$ in KB cells, through the inhibition of activity of p38 and extracellular signal-regulated kinases pathway $[19,29]$. In recent years, more and more researchers have found that oxidative stress promotes the secondary injury after $\mathrm{TB} \mathrm{I}$, and among multiple factors, nicotinamide adenine dinucleotide phosphate oxidase (NOX) is critical in regulating the production of ROS [1]. NOX2 in the main producer of ROS in brain tissue, a study has shown that activation of NOX2 is also closely related to the levels of $\beta$-amyloid and degeneration of dopaminergic neurons [5]. Besides, platelet, endothelial cells and lymphocytes also contribute to the development of the central nervous system, activation of these factors leads to the neurodegenerative process via inducing the production of amyloid precursor protein (APP) and $\beta$-amyloid peptide $(A \beta)$ in the cerebral arterial district, which is also closely related to the activation of NOX2 [32]. Furthermore, an increased expression of NOX2 promotes the activation of microglia surrounding $A \beta$-laden capillaries [6]. Another study using Huntington's disease mice model has shown that NOX2 is significantly activated in cortical and striatal synaptosomes as well as primary neurons derived from mice [31]. A previous study found that NOX enzymes contribute to the physiological function of brain [20], more and more evidence has shown that activation of NOX is a harmful factor for TBI patients, and exacerbate the primary injury [17], and has been regarded as a promising therapeutic target for TBI. In this study, we firstly proved that treatment of aloin could promote the recovery of brain function of mice after TBI, and further that inhibition of NOX2 combined with aloin treatment significantly increased the viability of cells with inhibition of the cellular apoptosis process. A further study found that inhibition of NOX2 combined with aloin treatment decreased the expression and secretion of pro-inflammatory factors. And these effects might be mediated by activation of the $\mathrm{PI} 3 \mathrm{~K} / \mathrm{AKT} / \mathrm{mTOR}$ signalling pathway.

\section{Material and methods Vector construction}

The CDNA of NOX2 was obtained with the following primer: Forward: 5'-TGTTTTCATTTCCTCATCAGAAG-3', Reverse: 5'-CCAACCACACCAGAATGACA-3' using PCR method (CW0741, CWBio). Primers and pcDNA-3.1-blank vector were digested with $\mathrm{Kpnl}$ (R3142S, NEB) and BamHI (R3136S, NEB). The pcDNA3.1-NOX2 overexpression vector was constructed using T4 polynucleotide kinase (M0201S, NEB). After construction, the vector was transfected into PC12 cells using Lipofectamine 3000 transfection reagent (L3000001, Thermo), and stable expressed cells were screened using 800 mg/ml G418 (10131027, Thermo).

\section{Cell culture and grouping}

PC12 (CRL-1721) cells were purchased from the American type culture collection (ATCC). Cells were cultured in RPMI-1640 medium supplied with 10\% fetal bovine serum (FBS) under a $37^{\circ} \mathrm{C}$ atmosphere with $5 \% \mathrm{CO}_{2}$. Cells were seeded into a 6-well plate in order to construct the TBI model in PC12 cells. Briefly, cells were scratched with a sterile plastic needle using a $9 \times 9$ square grid for $24 \mathrm{~h}$ according to the previous study [18]. Then, cells were divided into four groups: model group (CG), single aloin treatment group $(\mathrm{IG})$, aloin treatment combined with GSK2795039 treatment group (II) and aloin treatment combined with NOX2 overexpression group (IO). In aloin (HY-N0123, MCE) treatment group, cells were treated with $40 \mathrm{mg} / \mathrm{ml}$ aloin for $4 \mathrm{~h}$ [11], and in GSK2795039 treatment group, cells were treated with $25 \mu \mathrm{M}$ GSK2795039 (HY-18950, MCE) for $24 \mathrm{~h}$.

\section{Mice model construction and grouping}

20 C57/BL mice were obtained from Guangdong medical laboratory animal centre. Mice were housed in a $22-24^{\circ} \mathrm{C}$ humidified atmosphere, and water and food were freely available. The TBI model in mice was constructed according to a previous study [36]. Mice were firstly anaesthetized with ketamine $(100 \mathrm{mg} / \mathrm{kg}) /$ xylazine $(10 \mathrm{mg} / \mathrm{kg})$ through an intra-peritoneal injection, and then the craniotomy was performed with a hand-held electrical drill (Dremel $10.8 \mathrm{~V}$ ) and then mice were restrained by a stereotaxic device and subjected to a 1.5-mm deep impact (velocity of $5 \mathrm{~m} / \mathrm{s}$ ) via a computer-controlled impactor device 
(LinMot-Talk 1100) (impactor diameter of $2 \mathrm{~mm}$ ). Mice were divided into two groups: model group (MG) and aloin treatment group (IT). In the aloin treatment group, mice were treated with $20 \mathrm{mg} / \mathrm{kg}$ for continuous 3 days [11].

\section{Ethical statement}

Experiments on animal models were performed under the guideline of the Declaration of Helsinki and the principles and procedures outlined in the National Institutes of Health Guide for the Care and Use of Animals. Experiments on animal models were performed in Anqiu People's Hospital and approved by the Health Animal Care and Use Committee of Anqiu People's Hospital.

\section{Behaviour tests}

In order to detect the effect of IGF-1 on brain function after TBI, the Morris water maze (MWM) tests were performed. MWM tests were performed in a circular tank with $122 \mathrm{~cm}$ diameter and divided into four quadrants. Tests were performed on continuous 5 days with 4 trials per day, starting from SE, S, NW and W direction. The escape latency was set as the time used in reaching the escape platform, the escape test was performed on the sixth day. Time of travel distance was set as the time that mice spent in the quadrant and the number of mice crossed the quadrant after forcing them to swim for $60 \mathrm{~s}$ after removing the escape platform.

\section{MTT assay}

MTT assay was performed according to the manufacturer's protocol (M6494, Thermo). Briefly, cells were seeded into a 96-well plate at a concentration of $10^{4} /$ well, then cells were grouped and treated as described above. Cells were incubated with MTT reagent $(5 \mathrm{mg} / \mathrm{ml})$ for $4 \mathrm{~h}$. The optical density was measured at $570 \mathrm{~nm}$ using Multiskan Sky Microplate Spectrophotometer (51119570, Thermo).

\section{Flow cytometry}

Flow cytometry was performed according to the manufacturer's protocol (CW2574, CWbio). Briefly, cells were firstly seeded into $100 \mathrm{~mm}$ plate and grouped as described above. Then, cells were digested with trypsin and incubated with binding buffer. After incubated with Annexin-V/FITC and PI, the apoptotic cells were detected using NovoCyte (Agilent $\mathrm{BIO})$.

\section{RNA extraction}

RNA extraction was performed according to the manufacturer's protocol (CW0560, CWBio). Briefly, cells were lysed with lysis buffer and incubated at room temperature for 5 min. 70\% ethanol was added into the tube after centrifugation $(12,000 \mathrm{rpm}$ for $5 \mathrm{~min}$ ) and then collected into the absorption tube. After washing with washing buffer, RNA samples were eluted from the tube with elution buffer. RNA samples were stored at $-80^{\circ} \mathrm{C}$ until performing the following experiments.

\section{Real-time quantitative polymerase chain reaction)}

Reverse transcription and quantitative polymerase chain reaction ( $\mathrm{qPCR}$ ) was performed according to the manufacturer's protocol (CW0659, CWBio). Briefly, the reaction mixture was composed as recommended, and the reaction was performed with the following steps: reverse transcription at $45^{\circ} \mathrm{C}$ for $10 \mathrm{~min}$, pre-denaturation at $95^{\circ} \mathrm{C}$ for $5 \mathrm{~min}$, denaturation at $95^{\circ} \mathrm{C}$ for $10 \mathrm{~s}$ with these two steps repeated for 40 cycles: denaturation at $95^{\circ} \mathrm{C}$ for $10 \mathrm{~s}$, annealing at $60^{\circ} \mathrm{C}$ for $45 \mathrm{~s}$. Primers used in qPCR were listed: IL-1 $\beta$ : Forward: 5'-TGCCACCTTTTGACAGTGATG-3', Reverse: 5'-ATGTGCTGCTGCGAGATTTG-3'. Monocyte chemoattractant protein-1 (MCP-1): Forward: 5'-GGCTGGAGAGCTACAAGAGG-3', 5'-CTTGGTGACAAAAACTACAGC-3'; IL-10: Forward: 5'-GCGCTGTCATCGATTTCTCC-3', Reverse: 5'-CTCTTCACCTGCTCCACTGC-3'; IL-6: Forward: 5'-TCCTCTCTGCAAGAGACTTCC-3', Reverse: 5'-TTGTGAAGTAGGGAAGGCCG-3'. The expression of each target gene was determined using the $2-\Delta \Delta \mathrm{Cq}$ method [16].

\section{Protein extraction and western blotting analysis}

Cells were seeded into a $100 \mathrm{~mm}$ plate and grouped as described above. Then, cells were lysed with RIPA buffer and proteins were collected after centrifugation at 12,000 rpm for $10 \mathrm{~min}$. The concentration of protein samples were measured using BCA assay. $60 \mu \mathrm{g}$ protein samples were separated using $10 \%$ SDS-PAGE and then transferred onto a PVDF membrane. Membranes were blocked with skim milk for $1 \mathrm{~h}$ at room temperature, followed with incuba- 
tion with primary antibodies overnight at $4^{\circ} \mathrm{C}$ (AntiAKT (ab179463), p-AKT (ab38449), mTOR (ab2732), p-mTOR (ab109268), HIF-1 (ab51608), cleaved PARP1 (ab32064), PARP1 (ab227244), NOX2 (ab129068), cytochrome c (ab90529), APAF1 (ab2000), Caspase-9 (ab202068), cleaved caspase-3 (ab2302), caspase-3 (ab13847) and GAPDH (ab8245) were purchased from Abcam) and incubated with secondary antibody for 1 $\mathrm{h}$ at room temperature. The grey value of protein was detected using chemiluminescent immunoassay.

\section{Enzyme linked immunosorbent assay (ELISA)}

Enzyme linked immunosorbent assay (ELISA) was performed according to the manufacturer's protocols (ab186029, ab113852, ab138881, ab202410, purchased from Abcam). Briefly, cells were seeded into $100 \mathrm{~mm}$ plate, and then cultured and grouped as described above. Then, the supernatant was collected and added into each well of a 96-well plate, incubated for $60 \mathrm{~min}$ at $37^{\circ} \mathrm{C}$. And after washing with washing buffer, samples were incubated with TMB solution for $10 \mathrm{~min}$ at $37^{\circ} \mathrm{C}$. The optical density was measured at $450 \mathrm{~nm}$ using Multiskan Sky Microplate Spectrophotometer (51119570, Thermo).

\section{Results}

\section{Effect of IGF-1 on brain function of mice}

The results of the behaviour test were shown in Figure 1. The average escape latency of mice in each group was $70.3 \pm 5.2,64.2 \pm 4.7,59.1 \pm 4.1,53.0 \pm 3.6$ and $48.4 \pm 3.3$ in the control group, and $68.5 \pm 4.9$,
$58.3 \pm 4.2,47.2 \pm 3.8,41.1 \pm 3.5$ and $35.2 \pm 2.9$ in the IGF-1 treatment group. The average number of platform crossed in these groups was $2.1 \pm 0.3$ and $4.0 \pm 0.6$. The time spent in the target quadrant in these groups was $18.3 \pm 2.7$ and $27.1 \pm 4.1$ s. These results showed that treatment of IGF-1 significantly improved the brain function of mice after TBI.

\section{Detection of proliferation of cells in each group}

Proliferation of cells was shown in Figure 2. The viability of cells in CG, IG, II and IO group was $100 \pm 9.6,116.3 \pm 13.3,152.2 \pm 17.4$ and $103.3 \pm 11.0$. The viability was significantly increased in the II group ( $p<0.05)$ compared with the CG and IG group. And the percentage of apoptotic cells in these groups was $36.2 \pm 6.3,23.1 \pm 4.5,8.3 \pm 1.6$ and $31.0 \pm 5.1$. The percentage of apoptotic cells was significantly decreased in the IG and II group $(p<0.05)$ compared with the CG group, and was significantly decreased in the II group $(p<0.05)$ compared with the IG group.

\section{Detection of inflammation related genes in cells}

As shown in Figure 3, the expression of IL-1 $\beta$ in the CG, IG, II and IO groups was $1.9 \pm 0.4,1.4 \pm 0.3$, $0.8 \pm 0.2$ and $1.6 \pm 0.4$, respectively. The expression of IL- 6 in these groups was $1.8 \pm 0.3,1.5 \pm 0.3$, $0.9 \pm 0.2$ and $1.7 \pm 0.3$. The expression of $\mathrm{IL}-10$ in these groups was $0.9 \pm 0.2,1.3 \pm 0.3,2.2 \pm 0.4$ and 1.0 \pm 0.2 . The concentration of MCP-1 in these groups
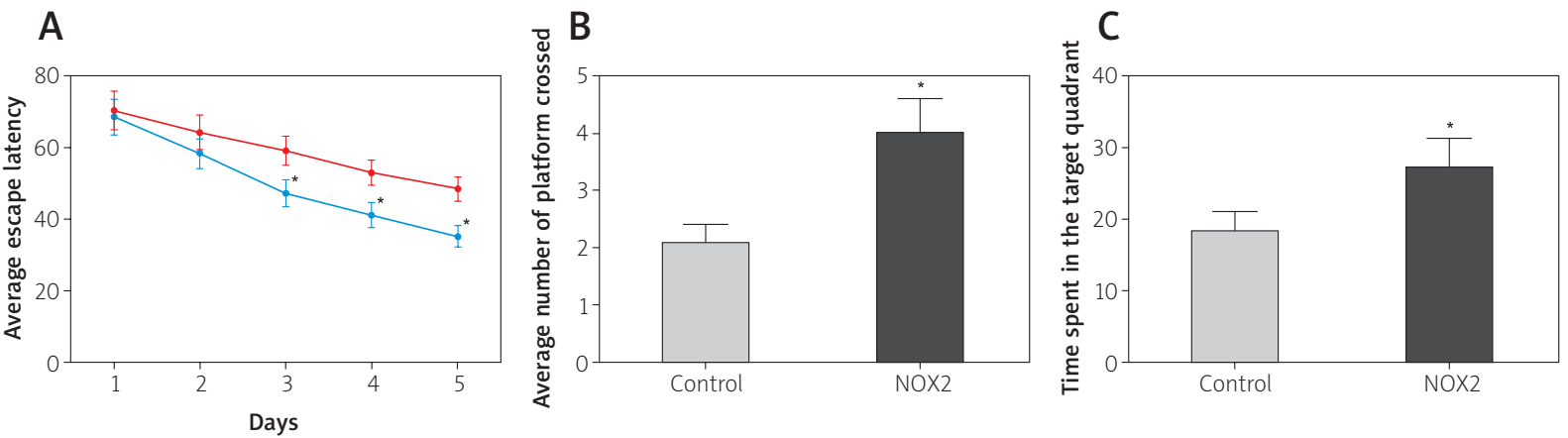

Fig. 1. Behaviour tests of the mice model with or without treatment of aloin. A) Average escape latency of the mice model on continuous 5 days. B) Average number of platform crossed of the mice model in each group. C) Time spent in the target quadrant of the mice model in each group. Each experiment was repeated three times independently. ${ }^{*} p<0.05$ compared with the control group. 
A

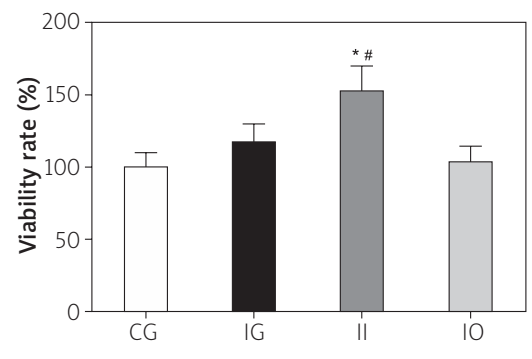

\section{B}

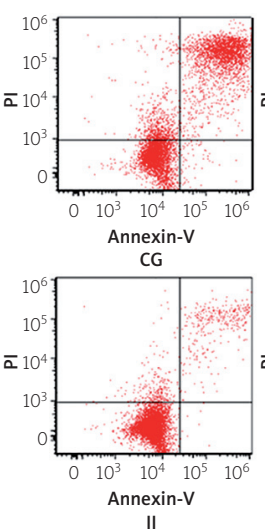

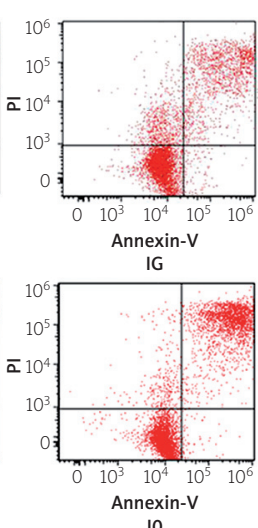

Fig. 2. Proliferation of cells in each group. A) Viability of cells in each group. B) Detection of apoptotic cells in each group. Each experiment was repeated three times independently. ${ }^{*} p<0.05$ compared with the CG group. ${ }^{*} p<0.05$ compared with the IG group.

A

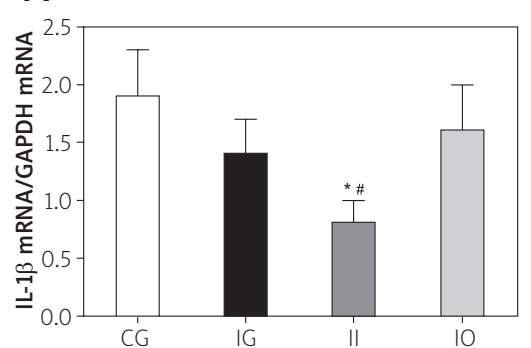

C

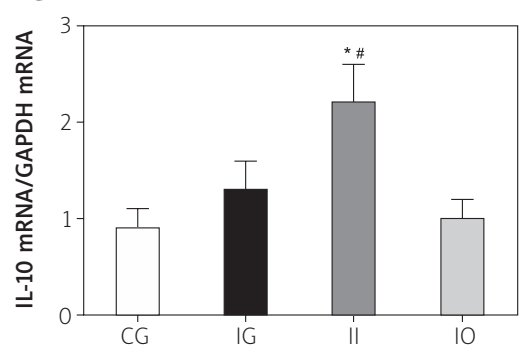

B

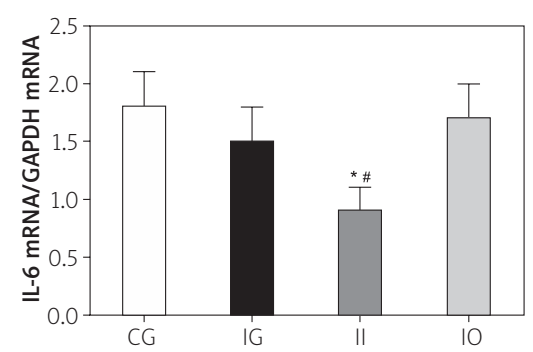

D

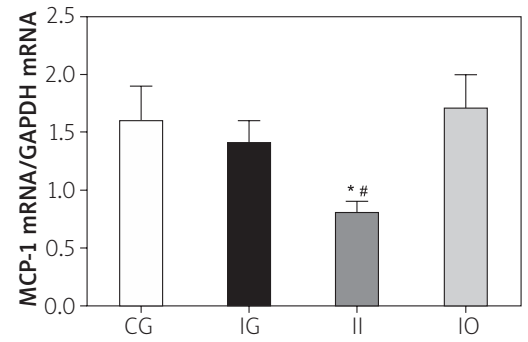

Fig. 3. Expression of interleukin (IL)-1 1 (A), IL-6 (B), IL-10 (C) and MCP-1 (D) in each group of the cell model. Each experiment was repeated three times independently. ${ }^{*} p<0.05$ compared with the CG group. ${ }^{*} p<0.05$ compared with the IG group. GAPDH was used as an internal control.

was $1.6 \pm 0.4,1.4 \pm 0.3,0.8 \pm 0.1$ and $1.7 \pm 0.5$. The expression of pro-inflammation factors was significantly decreased in the II group $(p<0.05)$ compared with the CG and IG group, while the expression of anti-inflammation factor was significantly increased in the II group $(p<0.05)$ compared with the CG and IG group.

\section{Expression of apoptosis related proteins in cells}

As shown in Figure 4, the expression of cyt $c$ in the CG, IG, II and IO groups of cells was $1.11 \pm 0.09$, $0.72 \pm 0.06,0.53 \pm 0.04$ and $0.91 \pm 0.07$, respectively. The expression of APAF1 in these groups was $0.91 \pm 0.07,0.71 \pm 0.05,0.42 \pm 0.03$ and $0.90 \pm 0.07$. 
A

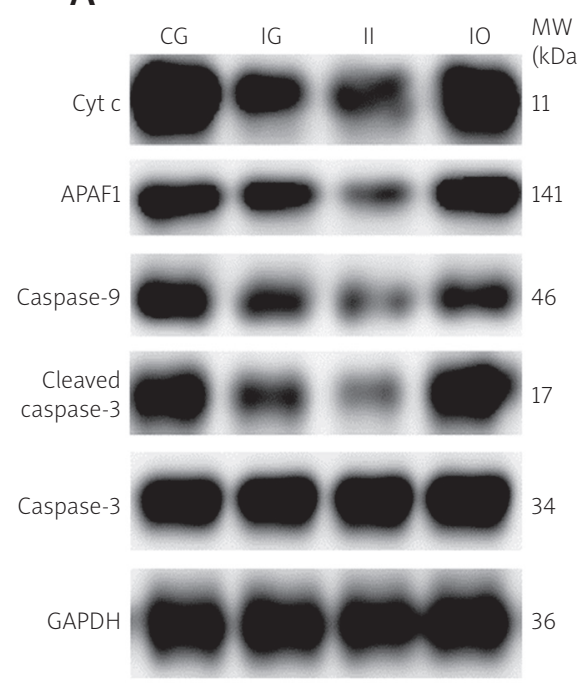

B
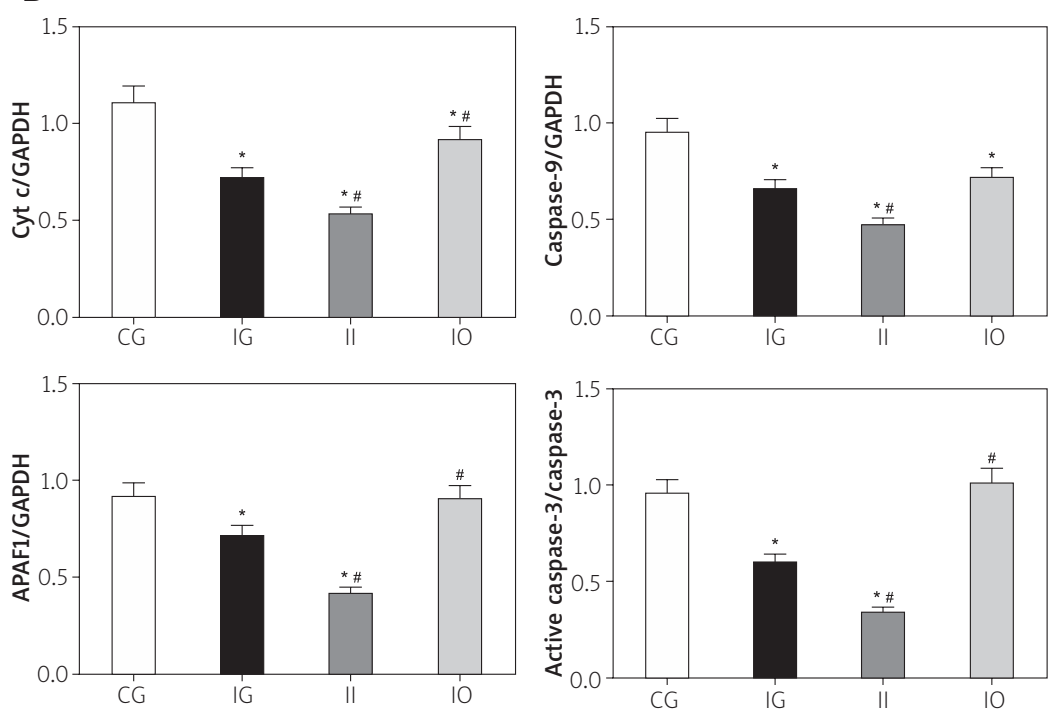

Fig. 4. Detection of apoptosis related proteins in each group of the cell model. A) Western blotting analysis of cyt c, APAF1, caspase-9, cleaved caspase-3 and caspase-3 in each group. B) Quantitative analysis of each target proteins. Each experiment was repeated three times independently. ${ }^{*} p<0.05$ compared with the CG group. ${ }^{*} p<0.05$ compared with the IG group. GAPDH was used as an internal control.

The expression of caspase- 9 in these groups was $0.95 \pm 0.07,0.66 \pm 0.05,0.47 \pm 0.04$ and $0.71 \pm 0.05$. The ratio of cleaved caspase-3/caspase- 3 in these groups was $0.96 \pm 0.07,0.60 \pm 0.05,0.34 \pm 0.03$ and $1.01 \pm 0.08$. The expression of pro-apoptosis proteins was significantly decreased in the II group compared with the CG and IG group $(p<0.05)$.

\section{Expression of the PI3K/AKT/mTOR signalling pathway}

As shown in Figure 5 , the ratio of p-AKT/AKT in the CG, IG, II and IO groups was $0.45 \pm 0.03,0.70 \pm 0.05$, $0.94 \pm 0.07$ and $0.45 \pm 0.03$, respectively. The ratio of p-mTOR/mTOR in these groups was $0.25 \pm 0.02$, $0.68 \pm 0.05,0.91 \pm 0.07$ and $0.60 \pm 0.05$. The expression of HIF- $1 \alpha$ in these groups was $0.84 \pm 0.06,0.85 \pm 0.07$, $1.05 \pm 0.08$ and $0.77 \pm 0.06$. The ratio of $p$-PARP/ PARP in these groups was $1.06 \pm 0.08,0.79 \pm 0.06$, $0.64 \pm 0.05$ and $1.05 \pm 0.08$. The expression of NOX 2 in these groups was $0.91 \pm 0.07,0.74 \pm 0.06,0.47 \pm 0.04$ and $0.95 \pm 0.07$. The PI3K/AKT/mTOR signalling pathway was significantly activated in the II group compared with the CG and IG group, while the expression of PARP and NOX2 was significantly decreased in the II group compared with the CG and IG group $(p<0.05)$.

\section{Concentration of inflammation related factors in culture medium of cells}

As shown in Figure 6, the concentration of superoxide dismutase 1 (SOD1) in culture medium of cells in the CG, IG, II and IO groups was $96.3 \pm 4.1,125.1 \pm 5.2$, $173.4 \pm 7.1$ and $103.2 \pm 4.6 \mathrm{ng} / \mathrm{ml}$, respectively. The ratio of GSH/GSSG in these groups was $1.0 \pm 0.2,1.3 \pm 0.3$, $2.2 \pm 0.5$ and $1.1 \pm 0.3$. The relative intensity of tetramethylrhodamine ethyl ester (TMRE) in these groups was $1.0 \pm 0.1,1.4 \pm 0.2,2.1 \pm 0.3$ and $1.1 \pm 0.2$. And the relative intensity of ROS in these groups was $1.0 \pm 0.2$, $0.7 \pm 0.1,0.3 \pm 0.1$ and $0.9 \pm 0.2$. The oxidative stress response was significantly inhibited in the II group compared with the CG and IG group $(p<0.05)$.

\section{Discussion}

Traumatic brain injury (TBI) is one of the leading causes of mortality and morbidity around the world, according to the previous study, with approximately 2.2 million emergency events, 300,000 hospitalizations and 52,000 deaths in the United States in 

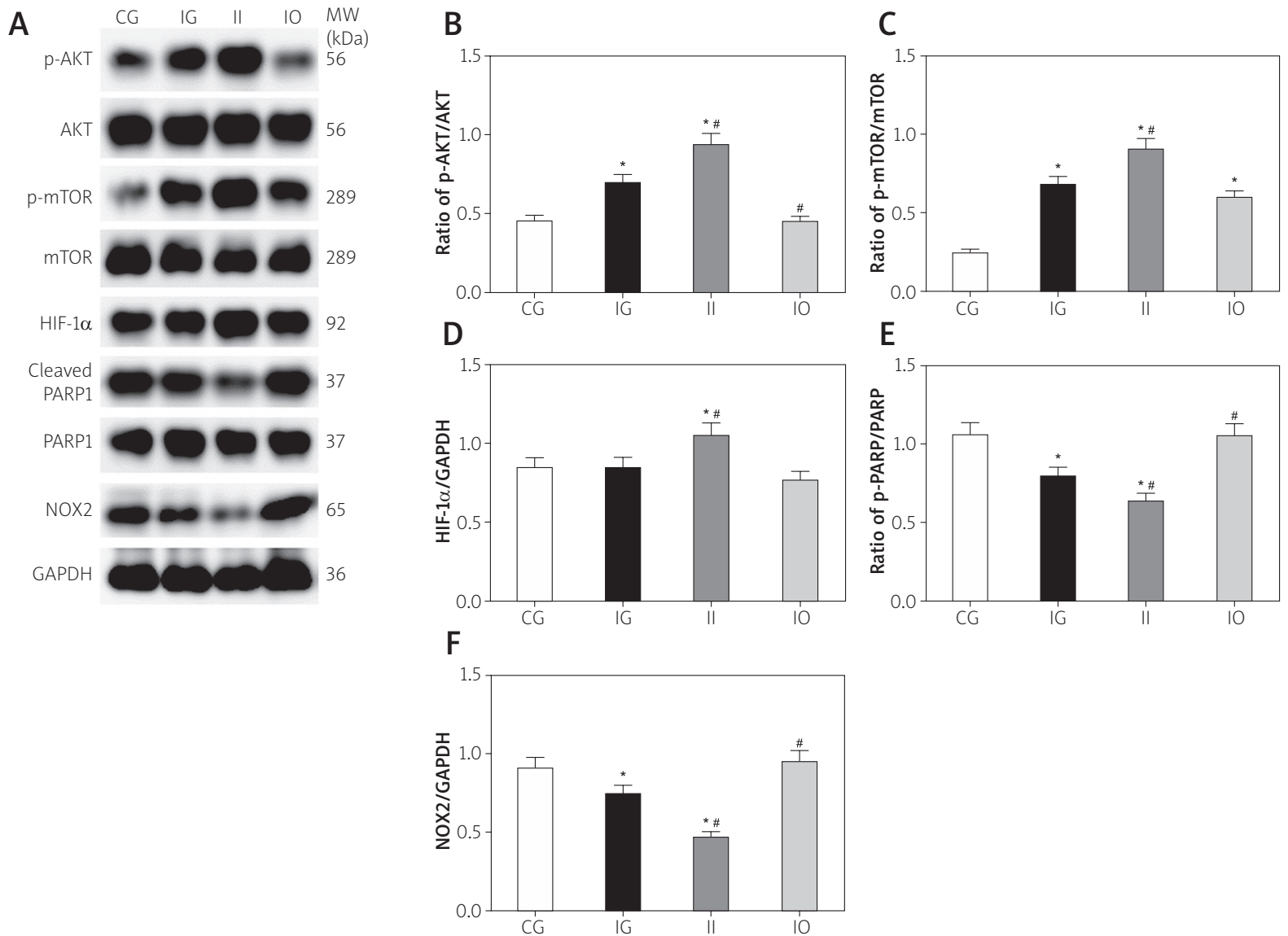

Fig. 5. Detection of key proteins in the PI3K/AKT/mTOR signalling pathway in each group of the cell model. A) Western blotting analysis of p-AKT, AKT, p-mTOR, mTOR, HIF-1 $\alpha$, cleaved PARP1, PARP1, NOX2 in each group of the cell model. B-F) Quantitative analysis of each target proteins. Each experiment was repeated three times independently. ${ }^{*} p<0.05$ compared with the CG group. ${ }^{*} p<0.05$ compared with the IG group. GAPDH was used as an internal control.

2010 [7]. Although lots of cost was spent each year on treatment of TBI, the successful treatment is still lacking.

Activation of the apoptotic process requires the activation of death activator proteins [37], among them, the release of cyt c from the mitochondria is the initial step of the apoptotic process [13]. To date, cyt $c$ has been regarded as a pro-apoptotic protein, and the release of cyt $c$ from mitochondrial membrane requires the oxidation of cardiolipin. After release to the cytosol, cyt $c$ interacts with the apoptotic protease activating factor-1 (Apaf-1) to form the apoptosome, which further activates caspases, leading to the degradation of cellular proteins [26]. The changes in conformation of apoptosome induce the binding of Apaf- 1 with cyt c, resulting in the recruitment and activation of caspase-9, further leading to the activation of caspase-3 [4]. These changes in apoptotic cells would lead to the reduction of mitochondrial membrane potential and generation of free radicals and nucleus condensation [27]. However, we noticed that cells under aloin combined with NOX2 inhibition treatment would reduce the activation of these proteins, which reflects the apoptosis process of cells after TBI was reduced, presented a significantly protective role.

Effective treatment for TBI is lacking nowadays, but recent studies have focused on the function of the AKT signalling pathway in the treatment of TBI. AKT, also known as protein kinase $B$, is regarded as a pro-survival pathway in multiple tissues including brain [33]. Using pharmacological inhibition of AKT, 
A

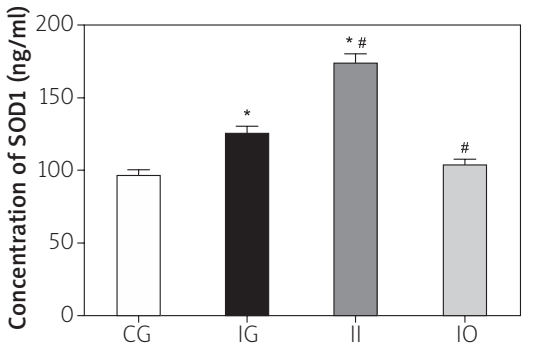

C

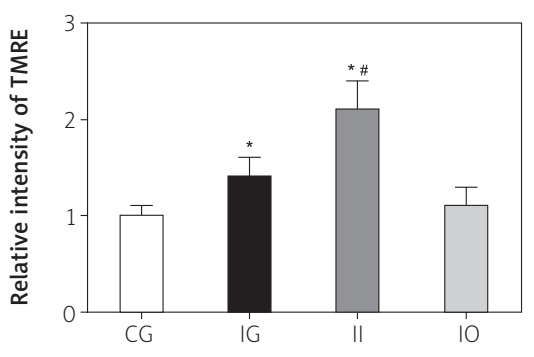

B

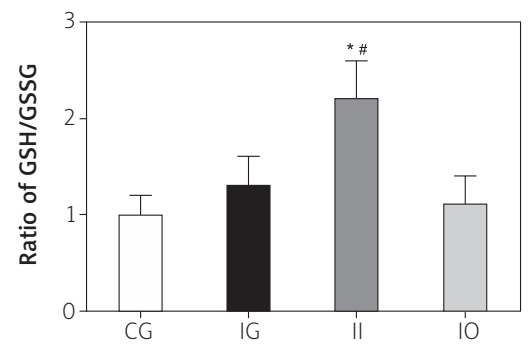

D

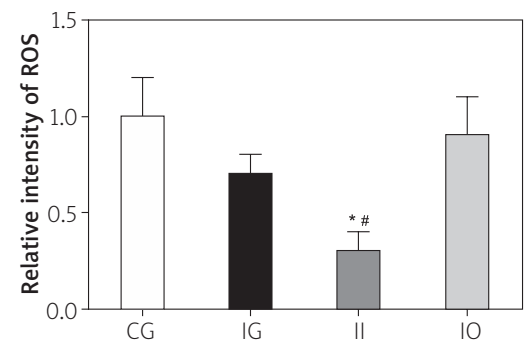

Fig. 6. Concentration of SOD1 (A), ratio of GSH/GSSG (B), relative intensity of TMRE (C) and ROS (D) in culture medium of the cell model in each group. Each experiment was repeated three times independently. ${ }^{*} p<0.05$ compared with the CG group. ${ }^{*} p<0.05$ compared with the IG group.

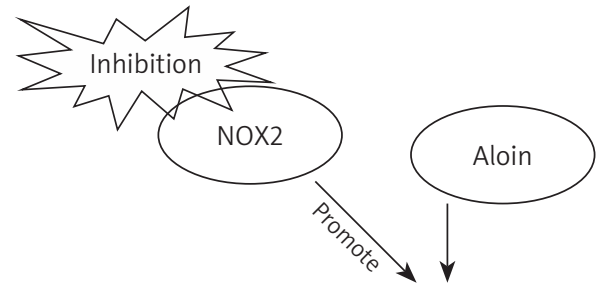

Inhibition of the PI3K/mTOR signalling pathway

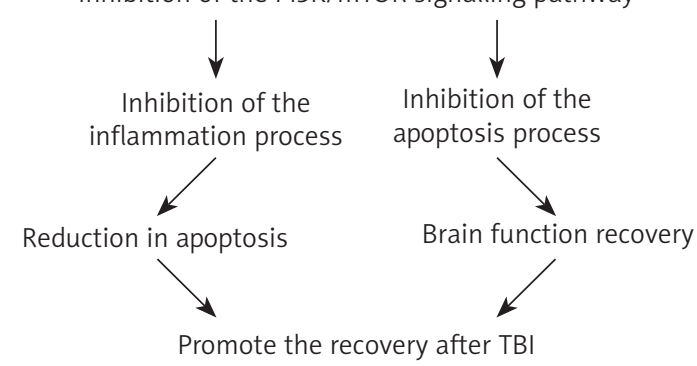

Fig. 7. Possible mechanism of NOX2 in promoting the therapeutic effect of aloin after TBI.

researchers found that the treatment is effective for $\mathrm{TBI}$, indicating the importance of AKT in treatment of TBI [35]. Besides, activation of AKT also attenuates the activation of the intrinsic apoptosis pathway, further reduces the activation of extensive cell death in neurons [38], and these effects leads to the reduction in post-injury lesion volume compared to the control group due to the inhibition of the intrinsic apoptosis pathway [30]. A downstream molecule of AKT, mTOR, was also activated after AKT activation, which further leads to the translation of HIF- $1 \alpha$ mRNA [14]. A previous study has shown that stimulation of mTOR promotes the stabilization and function of HIF-1 $\alpha$ [9]. Activation of the PI3K/AKTmTOR signalling pathway promotes the survival of cells and healing of wounds via regulation of multiple factors, including HIF-1 $\alpha$. HIF-1 $1 \alpha$ is a well-known transcription factor in response to hypoxia stimulation [39]. Activation of transcription factor binds with the hypoxiaresponse elements, leads to the activation of target genes, including HIF-1 $\alpha$. A previous study has shown that activation of HIF-1 $\alpha$ was observed after TBI, resulting in the reduction of tissue injury, performing a protective role, and this effect was lost after inhibition of HIF-1 $\alpha$ [35]. Besides anti-oxidative function, HIF-1 $\alpha$ also inhibits the glycolysis and fatty acid oxidation (FAO) through suppression of peroxisome proliferator-activated receptor- $\alpha$ (PPAR $\alpha$ ). A former study found that expression of PPAR $\alpha$ was elevated in NOX knockout mice, indicating that PPAR $\alpha$ might regulate the expression of NOXs through activation of HIF-1 $\alpha$. 
Downregulation of PPAR $\alpha$ reduced the infarct size and deposition of triglycerides after ischemia injury, suggesting that HIF- $1 \alpha$ presents a protective role via suppression of PPAR $\alpha$ and further inhibition of NOX2 or NOX4, resulting in reduction of ROS [23]. Here, the activation of the PI3K/AKT/HIF-1 $\alpha$ signalling pathway and suppression of PARP $\alpha$ were observed in the aloin combined with NOX2 inhibition group, showed that inhibition of NOX2 enhanced the therapeutic effect of aloin in TBI via inhibition of the cellular apoptotic process and inflammation process.

A previous study noticed that an early increase of NOX2 expression in specific brain areas promotes the development of non-pharmacologic and pharmacologic rodent models of psychosis [25]. Another study found that an increased expression of NOX2 was observed in cortical GABAergic neurons, which presented several pathological alterations [3] or changes in the distribution of calcium-binding protein in schizophrenia and bipolar disorder models [10]. The changes in expression of genes related to the inflammation and hypoxia were significantly reduced in $\mathrm{NOX2}^{-1-}$ animals, indicating that NOX2 promotes the inflammatory and hypoxia response in animal models [12]. These findings have shown that NOX2 contributes to the development of diseases, and alteration of NOX2 might be a therapeutic target in TBI and other diseases.

\section{Disclosure}

The authors declare no conflict of interest.

\section{References}

1. Altenhöfer S, Kleikers PW, Radermacher KA, Scheurer P, Rob Hermans JJ, Schiffers P, Ho H, Wingler K, Schmidt HH. The NOX toolbox: validating the role of NADPH oxidases in physiology and disease. Cell Mol Life Sci 2012; 69: 2327-2343.

2. Arosio B, Gagliano N, Fusaro LM, Parmeggiani L, Tagliabue J, Galetti P, De Castri D, Moscheni C, Annoni G. Aloe-Emodin quinone pretreatment reduces acute liver injury induced by carbon tetrachloride. Pharmacol Toxicol 2000; 87: 229-233.

3. Behrens MM, Ali SS, Dao DN, Lucero J, Shekhtman G, Quick KL, Dugan LL. Ketamine induced loss of phenotype of fast-spiking interneurons is mediated by NADPH-oxidase. Science 2007; 318: 1645-1647.

4. Bratton SB, Salvesen GS. Regulation of the Apaf-1-caspase-9 apoptosome. J Cell Sci 2010; 123 (Pt 19): 3209-3214.

5. Cahill-Smith S, Li JM. Oxidative stress, redox signaling and endothelial dysfunction in ageing-related neurodegenerative diseases: a role of NADPH oxidase 2. Br I Clin Pharmacol 2014; 78: 441-453.
6. Carrano A, Hoozemans JJ, van der Vies SM, Neuroinflammation and blood-brain barrier changes in capillary amyloid angiopathy. Neuroinflammation and blood-brain barrier changes in capillary amyloid angiopathy. Neurodegener Dis 2012; 10: 329-331.

7. Galgano M, Toshkezi G, Qiu X, Russell T, Chin L, Zhao LR. Traumatic brain injury: current treatment strategies and future endeavors. Cell Transplant 2017; 26: 1118-1130.

8. Hansson HA, Jennische E, Skottner A. Regenerating endothelial cells express insulin-like growth factor-I immunoreactivity after arterial injury. Cell Tissue Res 1987; 250: 499-505.

9. Hudson CC, Liu M, Chiang GG, Otterness DM, Loomis DC, Kaper F, Giaccia AJ, Abraham RT. Regulation of hypoxia-inducible factor 1alpha expression and function by the mammalian target of rapamycin. Mol Cell Biol 2002; 22: 7004-7014.

10. Jenkins TA, Harte MK, McKibben CE, Elliott JJ, Reynolds GP. Disturbances in social interaction occur along with pathophysiological deficits following subchronic phencyclidine administration in the rat. Behav Brain Res 2008; 194: 230-235.

11. Jing Y, Yang DX, Wang W, Yuan F, Chen H, Ding J, Geng Z, Tian HL. Aloin protects against blood-brain barrier damage after traumatic brain injury in mice. Neurosci Bull 2020; 36: 625-638.

12. Karim AS, Reese SR, Wilson NA, Jacobson LM, Zhong W, Djamali A. Nox2 is a mediator of ischemia reperfusion injury. Am J Transplant 2015; 15: 2888-2899.

13. Kulikov AV, Shilov ES, Mufazalov IA, Gogvadze V, Nedospasov SA, Zhivotovsky B. Cytochrome c: the Achilles' heel in apoptosis. Cell Mol Life Sci 2012; 69: 1787-1797.

14. Laughner E, Taghavi P, Chiles K, Mahon PC, Semenza GL. HER2 (neu) signaling increases the rate of hypoxia-inducible factor 1alpha (HIF-1alpha) synthesis: novel mechanism for HIF-1-mediated vascular endothelial growth factor expression. Mol Cell Biol 2001; 21: 3995-4004.

15. Li CY, Suzuki K, Hung YL, Yang MS, Yu CP, Lin SP, Hou YC, Fang SH. Aloe metabolites prevent LPS-induced sepsis and inflammatory response by inhibiting mitogen-activated protein kinase activation. Am J Chin Med 2017; 45: 847-861.

16. Livak KJ, Schmittgen TD. Analysis of relative gene expression data using real-time quantitative PCR and the 2(-Delta Delta $C(T)$ ) method. Methods 2001; 25: 402-408.

17. Ma MW, Wang J, Dhandapani KM, Wang R, Brann DW. NADPH oxidases in traumatic brain injury promising therapeutic targets? Redox Biol 2018; 16: 285-293.

18. Ma Y, Liu W, Wang Y, Chao X, Qu Y, Wang K, Fei Z. VEGF protects rat cortical neurons from mechanical trauma injury induced apoptosis via the MEK/ERK pathway. Brain Res Bull 2011; 86 : 441-446.

19. Na HS, Song YR, Kim S, Heo JY, Chung HY, Chung J. Aloin inhibits interleukin (IL)-1 $\beta$-stimulated IL-8 production in KB cells. J Periodontol 2016; 87: e108-115.

20. Nayernia Z, Jaquet $\mathrm{V}$, Krause KH. New insights on NOX enzymes in the central nervous system. Antioxid Redox Signal 2014; 20 : 2815-2837.

21. Nizamutdinov D, Shapiro LA. Overview of traumatic brain injury: an immunological context. Brain Sci 2017; 7: E11.

22. Olczak M, Poniatowski ŁA, Niderla-Bielińska J, Kwiatkowska M, Chutorański D, Tarka S, Wierzba-Bobrowicz T. Concentration of microtubule associated protein tau (MAPT) in urine and saliva 
as a potential biomarker of traumatic brain injury in relationship with blood-brain barrier disruption in postmortem examination. Forensic Sci Int 2019; 301: 28-36.

23. Poniatowski ŁA, Wojdasiewicz P, Krawczyk M, Szukiewicz D, Gasik R, Kubaszewski Ł, Kurkowska-Jastrzębska I. Analysis of the role of CX3CL1 (Fractalkine) and its receptor CX3CR1 in traumatic brain and spinal cord injury: insight into recent advances in actions of neurochemokine agents. Mol Neurobiol 2017; 54: 2167-2188.

24. Sánchez M, González-Burgos E, Iglesias I, Gómez-Serranillos MP. Pharmacological update properties of aloe vera and its major active constituents. Molecules 2020; 25: 1324.

25. Schiavone S, Jaquet V, Sorce S, Dubois-Dauphin M, Hultqvist M, Bäckdahl L, Holmdahl R, Colaianna M, Cuomo V, Trabace L, Krause $\mathrm{KH}$. NADPH oxidase elevations in pyramidal neurons drive psychosocial stressinduced neuropathology. Transl Psychiatry 2012; 2: e111.

26. Shakeri R, Kheirollahi A, Davoodi J. Apaf-1: Regulation and function in cell death. Biochimie 2017; 135: 111-125.

27. Shein NA, Horowitz M, Shohami E. Heat acclimation: a unique model of physiologically mediated global preconditioning against traumatic brain injury. Prog Brain Res 2007; 161: 353-363.

28. Taylor CA, Bell JM, Breiding MJ, Xu L. Traumatic brain injuryrelated emergency department visits, hospitalizations, and deaths - United States, 2007 and 2013. MMWR Surveill Summ 2017; 66: 1-16.

29. Thunyakitpisal P, Ruangpornvisuti V, Kengkwasing P, Chok

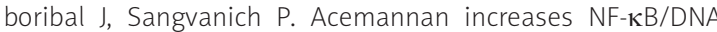
binding and IL-6/-8 expression by selectively binding Toll-like receptor-5 in human gingival fibroblasts. Carbohydr Polym 2017; 161: 149-157.

30. Umschweif G, Alexandrovich AG, Trembovler V, Horowitz M, Shohami E. Hypoxia-inducible factor 1 is essential for spontaneous recovery from traumatic brain injury and is a key mediator of heat acclimation induced neuroprotection. J Cereb Blood Flow Metab 2013; 33: 524-531.

31. Valencia A, Sapp E, Kimm JS, McClory H, Reeves PB, Alexander J, Ansong KA, Masso N, Frosch MP, Kegel KB, Li X, DiFiglia M. Elevated NADPH oxidase activity contributes to oxidative stress and cell death in Huntington's disease. Hum Mol Genet 2013; 22: 1112-1131.

32. Violi F, Loffredo L, Carnevale R, Pignatelli P, Pastori D. Athero thrombosis and oxidative stress: mechanisms and management in elderly. Antioxid Redox Signal 2017; 27: 1083-1124.

33. Wang L, Zhou K, Fu Z, Yu D, Huang H, Zang X, Mo X. Brain development and Akt signaling: the crossroads of signaling pathway and neurodevelopmental diseases. J Mol Neurosci 2017; 61: 379-384.

34. Wojdasiewicz P, Poniatowski ŁA, Turczyn P, Frasuńska J, Paradowska-Gorycka A, Tarnacka B. Significance of omega-3 fatty acids in the prophylaxis and treatment after spinal cord injury in rodent models. Mediators Inflamm 2020; 2020: 3164260.

35. Wu CC, Bratton SB. Regulation of the intrinsic apoptosis pathway by reactive oxygen species. Antioxid Redox Signal 2013; 19: 546-558.

36. Xu X, Yin D, Ren H, Gao W, Li F, Sun D, Wu Y, Zhou S, Lyu L, Yang M, Xiong J, Han L, Jiang R, Zhang J. Selective NLRP3 inflammasome inhibitor reduces neuroinflammation and improves long-term neurological outcomes in a murine model of traumatic brain injury. Neurobiol Dis 2018; 117: 15-27.

37. Zamaraev AV, Kopeina GS, Prokhorova EA, Zhivotovsky B, Lavrik IN. Post-translational modification of caspases: the other side of apoptosis regulation. Trends Cell Biol 2017; 27: 322-339.

38. Zhang X, Chen Y, Jenkins LW, Kochanek PM, Clark RS. Bench-tobedside review: Apoptosis/programmed cell death triggered by traumatic brain injury. Crit Care 2005; 9: 66-75.

39. Zhang L, Wang H. Targeting the NF-E2-related factor 2 pathway: a novel strategy for traumatic brain injury. Mol Neurobiol 2018; 55: $1773-1785$ 